\title{
Inhibitors of tyrosyl-DNA phosphodiesterase 1 (Tdp1) leelamine and berberine derivatives as prototypes of antitumor drugs
}

\author{
Mamontova E. ${ }^{1,2 *}$, Kovaleva K. ${ }^{2,3}$, Shirokova E. ${ }^{2,3}$, Zakharenko A. ${ }^{1}$, Yarovaya O. ${ }^{3}$, \\ Luzina O. ${ }^{3}$, Zakharova O. ${ }^{1}$, Salakhutdinov N. ${ }^{2,3}$, Lavrik O. ${ }^{1,2}$ \\ ${ }^{1}$ Institute of Chemical Biology and Fundamental Medicine, SB RAS, Novosibirsk, Russia \\ ${ }^{2}$ Novosibirsk State University, Novosibirsk, Russia \\ ${ }^{3}$ Institute of Organic Chemistry, SB RAS, Novosibirsk, Russia \\ *e-mail: evgeniya.mm.94@gmail.com
}

Key words: DNA repair, chemotherapy, tyrosyl-DNA phosphodiesterase 1, SCAN1, leelamine, berberine

Motivation and Aim: Tyrosyl-DNA phosphodiesterase 1 (Tdp1) is a promising target for cancer and neurodegenerative therapy. Tdp1 plays an important role in the removal of damage caused by topoisomerase 1 (Top1) inhibitors camptothecin and its derivatives topotecan and irinotecan. Last two compounds are the antitumor drugs widely used in clinical practice [1]. It makes this enzyme a promising target to enhance anticancer treatment in combination with DNA damaging therapies such as Top1 inhibitors. Moreover there is a natural mutant of Tdp1 which is responsible for the development of a neurodegenerative disease spinocerebellar ataxia with axonal neuropathy (SCAN1) [2]. The suppression of SCAN1 mutant activity can improve the SCAN1 patients' condition and prevent the progression of the disease. The natural compounds leelamine and berberine derivatives were chosen as potential Tdp1 and SCAN1 inhibitors based on their multiple biological activities.

Methods and Algorithms: To determine the inhibitory properties of compounds, fluorescent DNA biosensor was used [3]. $\mathrm{IC}_{50}$ values for each compound were calculated. To determine the own cytotoxicity of compounds and their cytotoxicity in combination with topotecan a standard MTT test was used.

Results: During the screening of 42 compounds 20 effective inhibitors of Tdp1 with $\mathrm{IC}_{50}$ values less than $1 \mu \mathrm{M}$ were found. The screening of 16 compounds against SCAN1 showed 10 inhibitors of SCAN1. Most of compounds appeared to be nontoxic for lung cancer A549 cell line. Two leelamine derivatives enhanced the cytotoxicity of topotecan. Conclusion: Both leelamine and berberine derivatives showed themselves as promising compounds for further development of anticancer drugs and drugs for treatment of the neurodegenerative disease SCAN1.

Acknowledgements: Supported by RFBR (grants 17-04-01071 and 18-33-00297) and by RSF (grant 19-13-00040).

\section{References}

1. Pommier Y. Topoisomerase I inhibitors: camptothecins and beyond. Nature Reviews. Cancer. 2006;6(10):789-802.

2. Takashima H. et al. Mutation of TDP1, encoding a topoisomerase I-dependent DNA damage repair enzyme, in spinocerebellar ataxia with axonal neuropathy. Nature Genetics. 2002;32(2):267-272.

3. Zakharenko A. et al. Synthesis and biological evaluation of novel tyrosyl-DNA phosphodiesterase 1 inhibitors with a benzopentathiepine moiety. Bioorganic Med. Chemistry. 2015;23(9):2044-2052. 